DRAFT VERSiON JANUARY 17, 2018

Preprint typeset using $\mathrm{IAT}_{\mathrm{E}} \mathrm{X}$ style emulateapj v. 12/16/11

\title{
PREDICTED NUMBER, MULTIPLICITY, AND ORBITAL DYNAMICS OF TESS M DWARF EXOPLANETS
}

\author{
SARAH BALLARD ${ }^{1,2}$ \\ Draft version January 17, 2018
}

\begin{abstract}
We present a study of the M dwarf exoplanetary systems forthcoming from NASA's TESS mission. While the mission's footprint is too complex to be characterized by a single detection completeness, we extract an ensemble completeness function that recovers the $\mathrm{M}$ dwarf planet detections from previous work. We employ this completeness function, together with a dual-population planet occurrence model that includes compact multiple planetary systems, to infer anew the planet yield. We predict both the number of M dwarf planets likely from TESS and their system architectures. We report four main findings: first, that TESS will likely detect more planets orbiting $\mathrm{M}$ dwarfs that previously predicted. Around stars with spectral types between M1V-M4V, we predict TESS will find $990 \pm 350$ planets orbiting $715 \pm 255$ stars, a 1.5-fold increase over previous predictions. Secondly, TESS will find two or more transiting planets around $20 \%$ of these host stars, a number similar to the multiplicity yield of NASA's Kepler mission. Thirdly, TESS light curves in which one or more planets are detected will often contain transits of additional planets below the detection threshold of TESS. Among a typical set of 200 TESS hosts to one or more detected planets, $116 \pm 28$ transiting planets will be missed. Transit follow-up efforts with the photometric sensitivity to detect an Earth or larger around a mid-M dwarf, even with very modest period completeness, will readily result in additional planet discoveries. And fourth, the strong preference of TESS for systems of compact multiples indicates that TESS planets will be dynamically cooler on average than Kepler planets, with $90 \%$ of TESS planets residing in orbits with $e<0.15$.

Subject headings: eclipses — stars: planetary systems
\end{abstract}

\section{INTRODUCTION}

NASA's TESS Mission (Ricker et al. 2014) will furnish the vast majority of small, rocky planets for atmospheric study. A typical TESS target star receives 27 days of continuous observation, so the sensitivity of the mission strongly favors short periods (Sullivan et al. 2015). A handful of transits of a small planet will be detectable over this duration only if those transits are individually large, which is why $75 \%$ of small planets detected by TESS are expected to orbit M dwarfs (Sullivan et al. 2015). In fact, it is likely that every small planet discovered by TESS to reside in its star's habitable zone will orbit an M dwarf (Sullivan et al. 2015). Combining this fact with the favorable signal-to-noise ratio of a planetary transmission spectrum around a small star (Tarter et al. 2007), M dwarfs will likely be the majority of sites for focused follow-up atmospheric study in the next decade with the James Webb Space Telescope (JWST, Gardner et al. 2006). The forthcoming TESS sample of planets orbiting M dwarfs will likely contain many targets of the first biosignature searches.

The ensemble of planets orbiting $M$ dwarfs has come into focus from a combination of radial velocity, microlensing, high-contrast imaging, and transit surveys (Bonfils et al. 2013; Johnson et al. 2010; Clanton \& Gaudi 2016; Montet et al. 2014; Bowler et al. 2015; Dressing \& Charbonneau 2013, 2015; Morton \& Swift 2014; Muirhead et al.|2015. For a detailed summary, see Shields et al.|2016). In particular,

\footnotetext{
${ }^{1}$ MIT Kavli Institute for Astrophysics \& Space Research, Cambridge, MA 02139, USA; sarahba@mit.edu

2 MIT Torres Fellow for Exoplanetary science
}

the photometric sensitivity of NASA's Kepler mission illuminated the population of planets smaller than $4 R_{\oplus}$ in orbit around $\mathrm{M}$ dwarfs, showing that they are more common around late spectral types than around FGK dwarfs (Howard et al. 2012; Mulders et al. 2015). They are so common, in fact, that Morton \& Swift (2014) found $2.00 \pm 0.45$ planets per $\mathrm{M}$ dwarfs, and Dressing \& Charbonneau (2015) reported a similar value of $2.5 \pm 0.2$ planets per star.

Yet, $\mathrm{M}$ dwarf planetary systems resist a simple, onepopulation explanation. The top panels of Figure [1 shows the result of using one mode of planet occurrence. Drawing 2-3 planets per star from the occurrence rate of Dressing \& Charbonneau (2015) furnishes only a fair fit to the Kepler properties of detected planets orbiting M dwarfs (Ballard \& Johnson 2016). One explanation is that the model of $2-3$ planets per star, with the underlying period and radius distribution in Dressing \& Charbonneau (2015), is in fact an average of two very different types of planetary systems. Observations of orbital eccentricity and spin-orbit alignment indicate that the systems with one transiting planet are dynamically distinct from those with two or more transiting planets. Planets in multiple-planet systems reside in more circular orbits (Xie et al. 2016), and are more aligned with the spins of their host stars (Morton \& Winn 2014). The number of transiting planets per star from Kepler also indicates two populations with different dynamical properties, one with at least 5 planets coplanar to within $2^{\circ}$, and other with 1-2 planet at larger orbital inclinations with respect to one another (Ballard \& Johnson 2016). This two-population model removes the discrepancy in the top left panel of 
Figure 11, in which the number of systems with only one transiting planet is underestimated, and the number of systems with two transiting planets is overestimated in equal measure. The two-population model also furnishes a better fit to other observables, like period, period ratio, and transit duration ratio (Dawson et al. 2016; Moriarty \& Ballard 2016). While the so-called "Kepler dichotomy" (Lissauer et al. 2011) explanation is not definitive, nor the only one (Gaidos et al. 2016; Bovaird \& Lineweaver 2017), we employ it here as a useful phenomenological descriptor of $\mathrm{M}$ dwarf planetary systems.

This two-mode model is also consistent with the independent measurement on the rate of "compact multiples": these are systems with at least two planets with orbital periods less than 10 days. Muirhead et al. (2015) showed that at least $20 \%$ of $\mathrm{M}$ dwarfs host a compact multiple system, and that fraction increases as stellar temperatures decreases. Within the two-population framework, these compact multiples are recognizable as the systems with $>5$ planets per star interior to 200 days. As we described above, compact multiples need to be included into order to reproduce the Kepler yield (Ballard \& Johnson 2016). Similarly, their inclusion should also result in a more realistic prediction of the TESS yield.

A sophisticated study of the likely TESS planet yield across the FGKM spectral types by Sullivan et al. (2015) incorporated the complicated TESS footprint, its instrumental limitations, the range of noise budgets within the surveyed stellar population, and false positive likelihoods. However, it employed the one-mode model of planet occurrence. The study undertaken by Muirhead et al. (2017) specific to the TESS M dwarfs accounted for additional complexities in the sample, particularly with respect to selecting exposure times. That study also assumed a single mode of planet occurrence. We hypothesize that an occurrence model that includes two distinct types of planetary systems, rather than one model that averages the the two, will result in a predicted TESS sample that will be different in important ways:

- The sample will contain more planet detections.

- It will find that TESS will detect two or more transiting planets around a substantial number of stars

- It will find that the TESS lightcurves with a detected planet will very often contain transits of additional planets lurking below the noise.

We do not aim to replicate the Sullivan et al. (2015) machinery in its complexity: because of that study, we already have an excellent understanding of how TESS will respond to incoming photons. Rather, we propose to extend the analysis for a different planet occurrence rate. In order to accomplish this, we need to extract the TESS completeness function for planets orbiting $M$ dwarfs as a function of radius and period. This function is not included in the Sullivan et al. (2015) study, but is readily derivable from it. Before we expand upon the result of Sullivan et al. (2015), we must demonstrate that we can replicate it, by showing that this completeness function correctly recovering the Sullivan et al. (2015) detections from their sample of injected planets . As for any survey, the TESS completeness function we will extract will be an average of the individual completenesses for each star observed by the mission.

With the TESS completeness in hand, we can apply it to a different sample of injected planets. We organize this study as follows: in Section 2, we describe our analysis, including the generation of synthetic planetary systems (§2.1) and how we create a mixture model of planetary systems $(\S 2.2)$. Section $\S 2.3$ describes the extraction of the $\mathrm{M}$ dwarf completeness function for TESS, and Section $\S 2.4$ describes how we apply it to the mixture model. Section 3 contains the results of this exercise. We enumerate the following goals for this study, which are addressed in the indicated sections.

1. Re-predict the number of planet detections among M dwarfs observed by TESS (§3.1)

2. Determine how often TESS will detect a single transiting planet, and how often it will detect two or more planets transiting the same star (§3.2)

3. Determine which additional planets, if any, will transit known TESS planet host stars but elude detection in TESS light curves ( $\$ 3.3)$

4. Predict the fraction of TESS detected systems that will have the "compact multiple" architecture, as compared to the underlying rate in nature ( 33.4$)$

5. Predict the eccentricity distribution of the detected TESS planets, and compare it to that of Kepler M dwarf planets $(\S 3.5)$

6. Approximate the number of planets TESS will detect that will exhibit transit-timing variations (TTV), using the rate of TTV occurrence measured by Kepler $(\S 3.6)$

7. Make a prediction for the bulk densities of planets detected by TESS, from planet formation theory. Compare these densities to the densities inferred for the Kepler planets. (§3.7)

In Section 4, we summarize our findings and conclude.

\section{ANALYSIS}

\subsection{Generating Planetary Systems}

To generate a realistic synthetic samples of planetary systems, we take the following steps. We draw periods and radii for each mock planetary system from the empirical distribution of Dressing \& Charbonneau (2015). We then employ the distributions of Limbach \& Turner (2015) to assign eccentricity. We assign planetary masses with the relations of Zeng \& Jacobsen (2017) for $R<$ $1.5 R_{\oplus}$ and Wolfgang et al. (2016) for $R>1.5 R_{\oplus}$. Rogers (2015) identified the cutoff between majority of rocky planets and a majority icy/gaseous planets at $1.5 R_{\oplus}$, but these two relations also naturally overlap at $1.5 R_{\oplus}$. We assess the stability of the system by ensuring that planets satisfy the criteron defined in Fabrycky et al. (2012b):

$$
\Delta \equiv\left(a_{2}-a_{1}\right) / R_{H_{1,2}}>2 \sqrt{3}
$$

where the mutual Hill Radius $R_{H_{1,2}}$ is defined by 

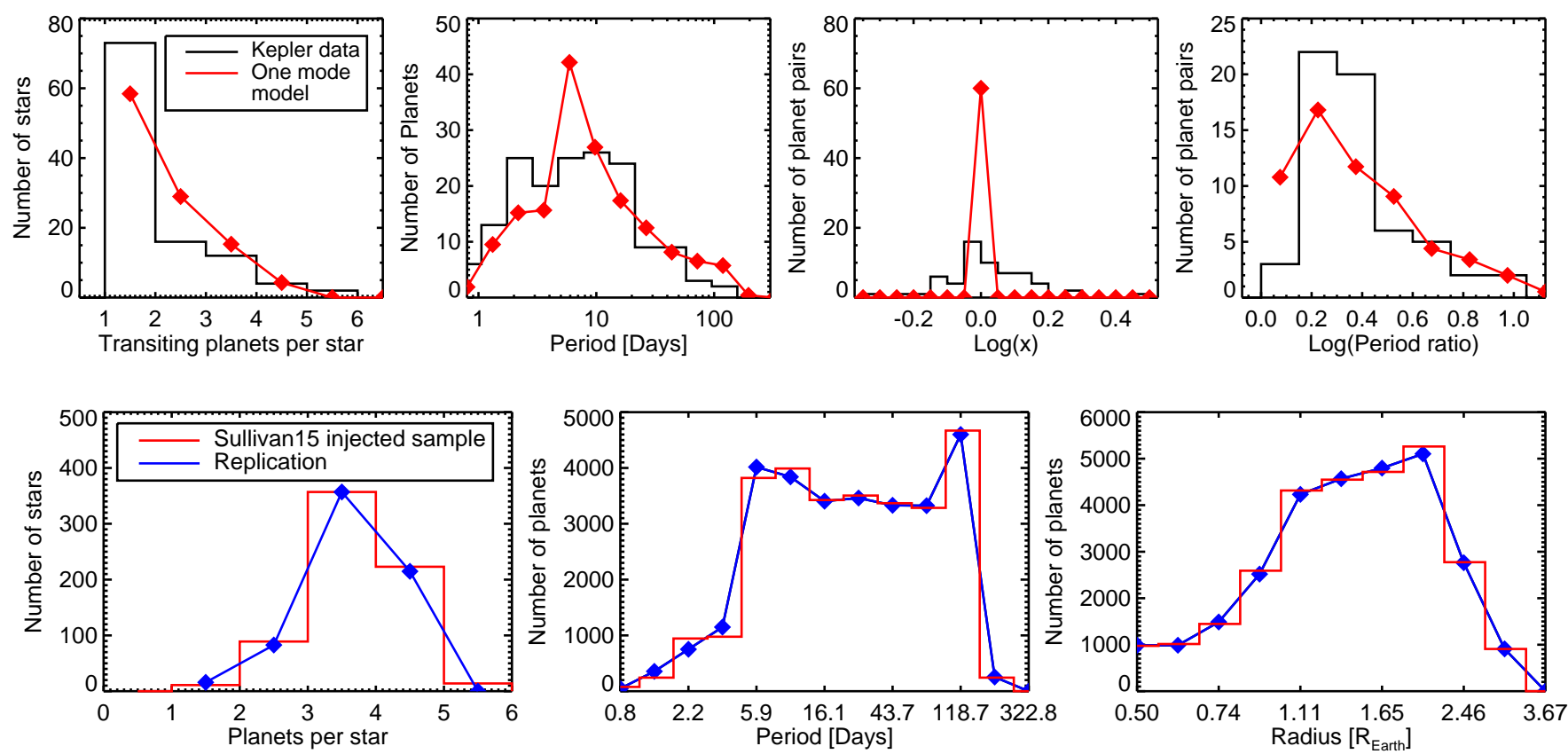

FIG. 1. - Top panels: Observed Kepler distributions (black) of detected planets in number of transiting planets star ${ }^{-1}$, period, transit duration ratio ( $\xi$ defined in Fabrycky et al. 2012b), and period ratio. Overplotted in red is the best one-mode planet occurrence model, with 2.5 planets per star drawn from Dressing \& Charbonneau (2015). The delta function in $\xi$ in the third panel is due to uniforming applying an orbital mutual inclination of zero degrees. Bottom panels: Consistency in the underlying distributions of number of planets per star, periods, and radii of our one-mode model (red) to the values used in Sullivan et al. (2015).

$$
R_{H_{1,2}}=\left[\frac{M_{1}+M_{2}}{3 M_{\star}}\right]^{1 / 3} \frac{\left(a_{1}+a_{2}\right)}{2} .
$$

This criterion is applicable for circular orbits. For eccentric orbits, we calculate the periapse and apoapse separation from the host star for each planet. We assume the orbits are stable if Equation 1 holds for the apoapse distance of the inner planet and the periapse of the outer planet. For generating synthetic TESS planetary systems, we employ a stellar mass of $0.4 M_{\oplus}$.

We then assign a boolean transit timing variation (TTV) flag to each transiting planet. Xie et al. (2014) showed that planets drawn from multi-transiting systems are likelier to exhibit TTVs, with that likeliness increasing as the number of transiting planets increases. We assign TTV probability per planet from that work, as defined by their "Case 3" (the most generous TTV occurrence rate): $3.5 \%$ per planets in singly-transiting systems, $7 \%$ for planets in doubly-transiting systems, $8 \%$ for planets in triply-transiting systems, and $10.4 \%$ for planets in systems with 4 or more transiting planets.

Finally, we calculate and record an independent density for each planet using only its mutual Hill spacing from neighboring planets. Dawson et al. (2016) predicted a theoretical relationship between these parameters: $\rho\left[\mathrm{g} / \mathrm{cm}^{3}\right]=(\Delta / 22)^{6}$, where $\Delta$ is defined in Equation 1. The scaling of density with mutual Hill separation provides a natural explanation, among others, for the fluffier nature of planets whose masses were measured with transit-timing variations (Wolfgang et al. 2016; Mills \& Mazeh 2017).

\subsection{Generating Mixture Models}

In the simplified "Kepler dichotomy" model, stars host one of two distinct types of planetary systems. Ballard \& Johnson (2016) showed that the Kepler M dwarf planets are well-described by one population of stars hosting flat and manifold systems of planets (with number of planets per star $N$ at least 5 , and orbital mutual inclinations $\sigma$ between 1 and $3^{\circ}$ ), with the other hosting 1 planet or 2 planets with high mutual inclination $\left(>8^{\circ}\right)$. Throughout this work, we refer to the former type of planetary system as "Population 1" or more descriptively as a "compact multiple". That work investigated the mixture specifically among detected planet hosts: in reality, the former type of planetary system is overly represented among detected planet hosts. This is because typical short periods within the multiple systems make it likelier that at least one planet will transit. The degree of this overrepresentation in both Kepler and TESS is discussed in Section 3.4 .

We define $N$ as the number of planets per star and $\sigma$ as the width of the Rayleigh distribution from which we draw their mutual inclinations. For a set of $\{N, \sigma\}$, we generate $10^{4}$ planetary systems using the criteria established in Section 2.1, employing the posterior distributions on this quantities from Ballard \& Johnson (2016). We then determine, based upon the assumption of random alignments for each planetary system with the line of sight, which planets transit their host star. We consider non-integer $N$ as follows. If a "typical" planetary system defined by $N$ and $\sigma$ hosts 3.5 planets, then $50 \%$ of stars host 3 planets (with eccentricities drawn from the CDF for 3-planet systems) and 50\% host 4 (with eccentricities drawn from the CDF for 4-planet systems).

The Fraction $f$ of stars in Population 1 in Ballard \& Johnson (2016) is the fraction of transiting 
systems in Population 1, not the fraction of stars. For each $f$, we now calculate an $f_{\star}$, the fraction of stars in Population 1 necessary to recover a contribution $f$ to the total number of transiting systems. For mixture models defined by the set $\left\{N_{1}, \sigma_{1}, N_{2}, \sigma_{2}, f_{\star}\right\}$, we randomly select $10^{4} \cdot f_{\star}$ stars populated by $\left\{N_{1}, \sigma_{1}\right\}$ and $10^{4} \cdot\left(1-f_{\star}\right)$ stars populated by $\left\{N_{2}, \sigma_{2}\right\}$. We then draw properties from the transiting planets among this final set of $10^{4}$ stars. Of course, the vast majority of these stars host zero transiting planets.

\subsection{Extracting the TESS Completeness Function}

To determine what a transit mission will detect, it is useful to know how often any given transiting planet will be detected, typically as a function of radius and orbital period. This quantity is the "completeness," and it is in principle distinct for every star the mission surveys. For example, when measuring Kepler's completeness to planets transiting FGK dwarfs, Christiansen et al. (2016) performed an injection-and-recovery exercise for each of 198,154 target stars. In absence of real light curves, Sullivan et al. (2015) used a signal-to-noise criterion to evaluate whether injected planets would be "detected" by TESS. The stare-and-step observation strategy of TESS means that most stars in the mission footprint receive 27 days of continuous photometry. However, overlap between observing fields results in some stars residing for up to a year in the field of view. Sullivan et al. (2015) study incorporated the full complexity of the TESS footprint and its stellar sample. The completeness of the survey to planets of a given size and period is not included in the study, but is derivable from it.

We first create a sample of injected planets, using the criteria described in Sullivan et al. (2015). This process is similar to the one described in Section 2.1 for a single mode of planet occurrence, except that (1) they assign more than one planet to a given star with independent probability, rather than assigning the number of planets per star a priori and (2) they assume a mutual inclination between orbits of zero. In the bottom panels of Figure 1, we show consistency between the sample we generated from the stated criteria and the one employed in that study. We aim now to find the completeness function that winnows this injected sample to the detected sample reported by Sullivan et al. (2015). That sample was generated at fixed resolution in both $\log$ (period) and $\log$ (radius) (inherited from Howard et al. 2012, Dressing \& Charbonneau 2013, and others), with approximate spacing of 1 dex between adjacent $\log$ (period) bins, and 0.2 dex between adjacent $\log$ (radius) bins. Because we cannot expect to extract information at a higher resolution than these values, we adopt their spacing to create a grid in $\log$ (period) and $\log$ (radius) space. We treat each individual bin at this resolution as a bucket that holds an integer number of planet detections. We calculate the number of detected planets from Sullivan et al. (2015) in each bin. We call this $D_{i, j}$, where $i$ is the index of the period bin and $j$ the index of the radius bin. In practice, the index $i$ spans periods from 0.8 to 320 days, in 13 regular log intervals of 1 dex, and the index $j$ spans radii from 0.3 to $4 R_{\oplus}$, in 17 regular log intervals of 0.2 dex. Similarly, we calculate the number of injected planets in each bin, $N_{i, j}$.
The actual surfaces of both $D_{i, j}$ and $N_{i, j}$ are shown in Figure 2. We multiply the number of injected planets in each bin by the completeness corresponding to that bin to produce the model number of detections in each bin, $\mu_{i, j}$.

We experimented with various functional forms for the completeness, which we call $C$. We adopt a smooth, analytic function for $C$, which we evaluate at the same resolution in $\log$ (period) and $\log$ (radius) to produce the completeness of each bin $C_{i, j}$. Trial verions of $C$ included a single-to-noise scaling, as well as simple power laws in $\log$ period or radius. Neither of the two functions for completeness, when applied to the injected planets, correctly approximated the number of detected planets: for example, while the predicted number of shortperiod planets might match, long-period planets would be strongly underestimated. We elected to use a polynomial in $\log$ (radius) and $\log$ (period) for $C$, with some constraints.

First, we require the completeness to be separable in period and radius (that is, $C\left(P, R_{p}\right)=C(P) \cdot C\left(R_{p}\right)$. We require that it be bounded between 0 and 1 . And we require it to be monotonic (increasing with radius and decreasing with period). Any monotonic polynomial can be modeled as a series of polynomials of the form Hawkins (1994):

$$
G(x)=a_{4}+a_{1} \int_{0}^{x} \prod_{i=1}^{K}\left[1+2 a_{2, i} t+\left(a_{2, i}^{2}+a_{3, i}^{2}\right) t^{2}\right] d t
$$

where all coefficients $a$ are all real numbers. Finding the optimal coefficients for a necessarily monotonic fit is a nonlinear problem. We first simplify it by using only the first term in the series, so that the completeness in both period and in radius have the form:

$$
C(x)=a_{4}+a_{1} x+a_{1} a_{2} x^{2}+\frac{a_{1}}{3}\left(a_{2}^{2}+a_{3}^{2}\right) x^{3} .
$$

This results in a total of 8 free parameters: 4 coefficients for the completeness with orbital period $C(P)$, and four for the completeness with planet radius $C\left(R_{p}\right)$. There does exist a linear algebra solution for finding $\left[a_{1}, a_{2}, a_{3}, a_{4}\right]$ for each polynomial, a modified version of singular value decomposition adapted for monotonic polynomials that relies upon Lagrange multipliers (Hawkins 1994; Murrav et al. 2013). The MonoPoly package in R (Turlach \& Murray 2016) implements those tools, which we used as a first estimate for the coefficients a: 4 for $C(P)$ and 4 for $C\left(R_{p}\right)$.

Poisson counting statistics describe integer numbers of transiting planets, so we evaluate the likelihood of $a$ with a Poisson likelihood function. This likelihood is conditioned on the "observed" number of planets detected in that bin $D_{i, j}$. The model number of planet detected in each bin $\mu_{i, j}(a)$ is dependent upon $a$ as follows:

$$
\mu_{i, j}(a)=N_{i, j} \cdot C_{i, j}(a),
$$

where $N_{i, j}$ is the number of injected planets in that bin and $C_{i, j}(a)$ is the completeness polynomial with coefficients $a$, evaluated at each bin. Figure 2 shows the surfaces of $D_{i, j}, N_{i, j}$, and $C_{i, j}(a)$ for a set of $a$. In the 
top panel, we show the detected planets published by Sullivan et al. (2015),$D_{i, j}$. The second panel shows our replication of the sample of the injected planets $N_{i, j}$. And the third panel shows a typical completeness $C_{i, j}$.
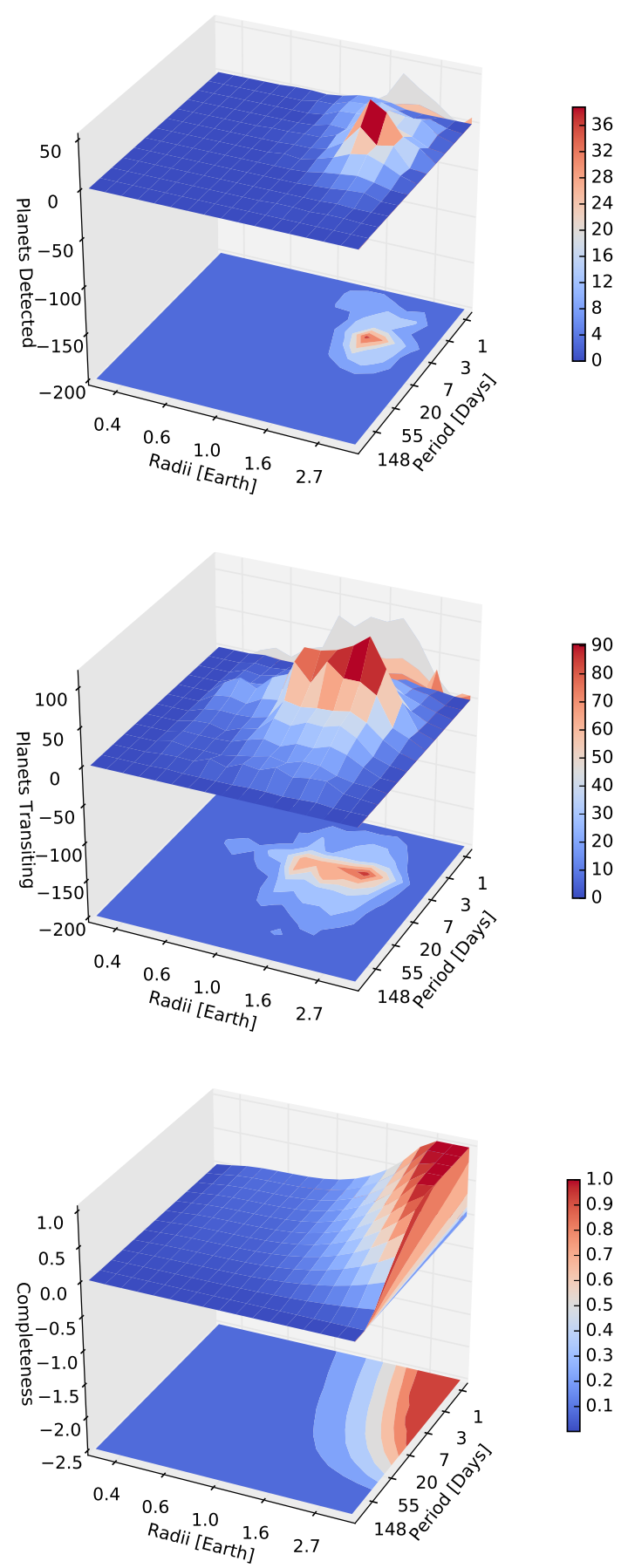

FIG. 2.- Top panel: Detected planets $D_{i, j}$ from Sullivan et al. (2015). Middle panel: Injected transiting planets sample generated using the criteria from same, $N_{i, j}$. Bottom panel: Typical polynomial completeness function evaluated at each bin. $C_{i, j}$.

The final Poisson likelihood function is defined as follows:

$$
\mathscr{L} \propto \prod_{i} \prod_{j} \frac{\mu_{i, j}(a)^{D_{i, j}} e^{-\mu_{i, j}(a)}}{D_{i, j} !} .
$$

We employ the Bayesian sampler MultiNest (Feroz \& Hobson 2008; Feroz et al. 2009, 2013, with Python implementation by Buchner et al. 2014) to evaluate these likelihoods and posterior distributions. In practice, MultiNest calculates the log of the likelihood defined in Equation 6. We use uniform priors for each of the polynomial coefficients, allowing them to vary to within $200 \%$ of the least-squares value. We enforce a monotonically decreasing polynomial in log period and a monotonically increasing polynomial in log radius, by setting the log likelihood to an arbitrarily low value otherwise $\left(-10^{-30}\right.$, in our case, in comparison to a typical log likelihood of -300). Figure 3 shows the posterior distributions for all 8 coefficients, with the least-squares values overplotted in blue. In 6 of 8 cases, the least-squares value lies within the $1 \sigma$ contour of the posterior distribution, and within $2 \sigma$ in all cases.

Figure 4s summarizes the results of the completeness fit. The injected planets $N_{i, j}$ are shown in black as a function of radius (top panel) and period (bottom panel). The detected planets $D_{i, j}$ are shown in red. Blue shows the completeness functions that best recovers $D_{i, j}$ from $N_{i, j}$, which we drawing from the posterior distribution in the coefficients $a$. The right-hand panels show the 1 and $2 \sigma$ confidence intervals on the model number of detected planets $\mu_{i, j}$ in grey. We verify that the extracted completeness is successful at recovering the detected planets from the injected planets of Sullivan et al. (2015). We note the large uncertainty of the completeness function at long periods: at 100 days, for example, completenesses of both $40 \%$ and $0 \%$ are consistent at $2 \sigma$ confidence. This is due to the inherent Poisson noisiness of only a few $(<10$ planets $)$ detections with which to constrain the completeness.

\subsection{Applying Completeness to Occurrence Mixture Model}

With a TESS completeness function in hand, we can apply it to a new sample of simulated transiting planets, this time employing the mixture model in Ballard \& Johnson (2016). As described in Section 2.1. we use the posteriors in the number of planets in both systems, $N_{1}$ and $N_{2}$, their average mutual inclinations $\sigma_{1}$ and $\sigma_{2}$, and the fraction of host stars in the first population $f$ directly from that work. The completeness coefficient posteriors in Figure 3 show that the coefficients are highly correlated. We cannot draw independently from their posterior distributions anew to sample the completeness. Rather, we save the completeness surface at each iteration of the MCMC chain.

For each transiting planetary system, we draw randomly from the sample of completeness surfaces $C_{i, j}$. For each individual planet's period and radius, we evaluate the detection likelihood from the completeness value corresponding to that bin. We take one additional step to enforce consistency for planets orbiting the same star. A joint random draw of detection probabilities for multiple planets can occasionally result in the nonsensical scenario of less-likely planets being detected, while more 

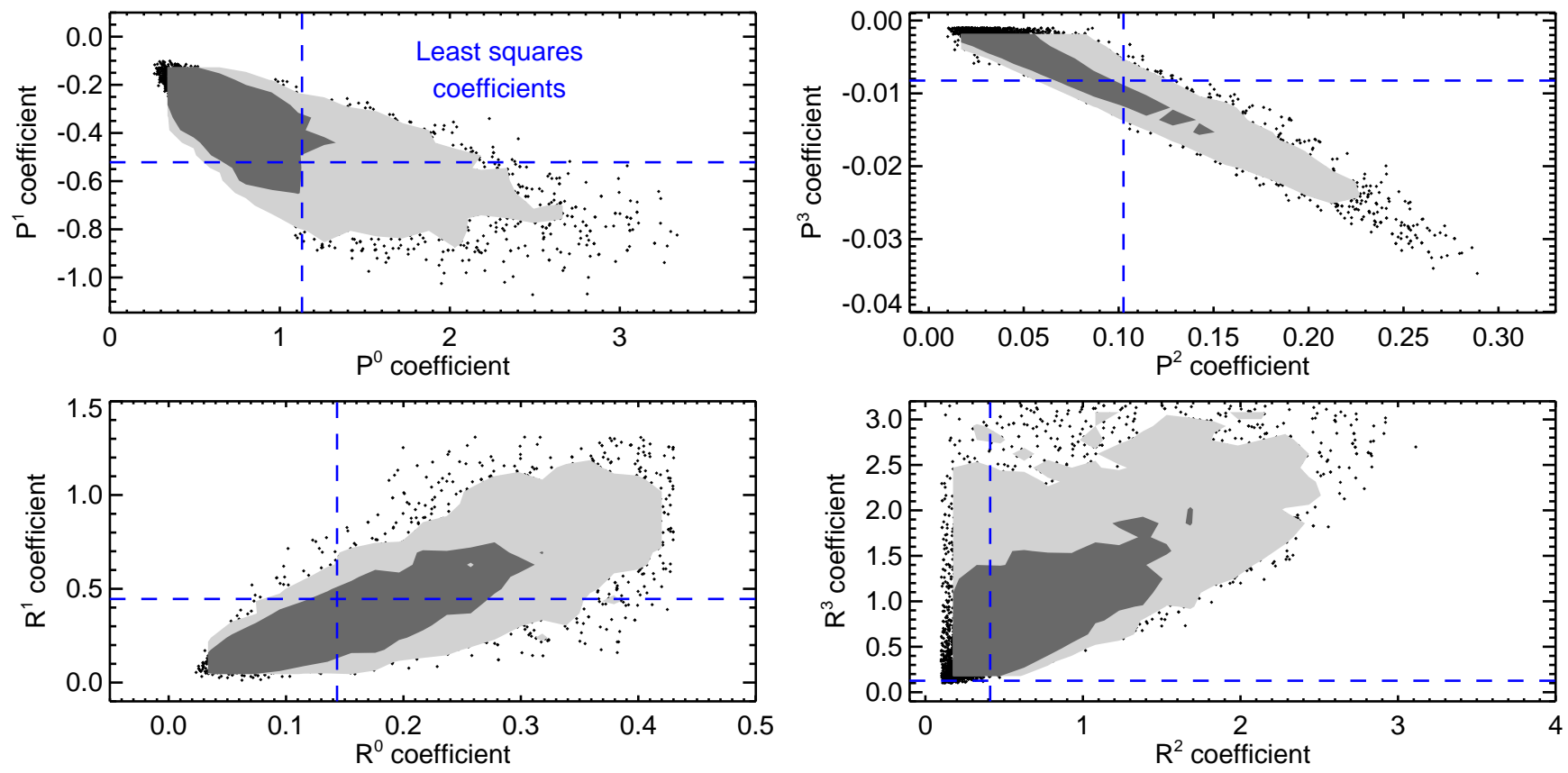

FIG. 3.- Posterior distributions for completeness function coefficients. Top two panels show the four coefficients for (log) period completeness (the coefficient for $P^{2}$ is indicated by the axis labeled $P^{2}$ ), while bottom two panels show coefficients for (log) radius completeness. Blue dotted lines show least-squares solution coefficients for a monotonic polynomial fit.
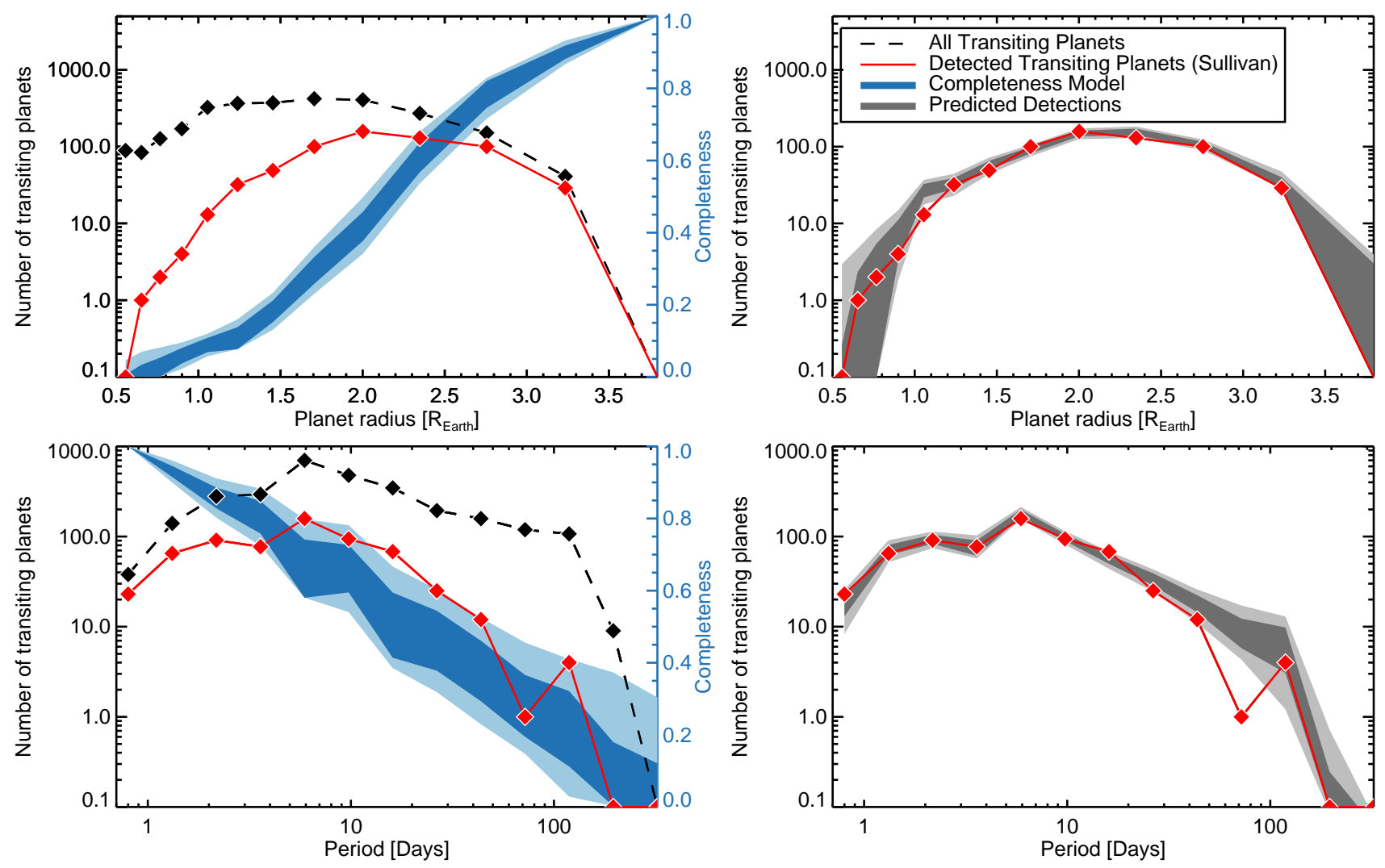

FIG. 4.- Left panels: Injected transiting planets $N$ (black) and detected planets $D$ (red) from Sullivan et al. (2015), as function of radius and period. Model completeness functions in radius and period are overplotted in dark $(1 \sigma)$ and light $(2 \sigma)$ blue: the right axis corresponds to completeness. Right panels: The population of detected Sullivan et al. (2015) planets, now with models $\mu$ for predicted planet detections overplotted in gray. 
"detectable" planets are missed. We take as an example a system of two transiting planets: Planet 1 with a period and radius assigning it a 50\% detection probability and Planet 2 with a radius and period assigning it a $5 \%$ detection probability. Out of 200 draws for a set of two random numbers between 0 and 1 , there will be 5 instances in which Planet 2 is detected and Planet 1 is missed. This is a sensible scenario for an ensemble of stars, but for planets orbiting the same star, we consider the SNR ratios of the two planets (assume the same noise budget for both), and if the missed planet has a higher SNR than the detected planet, we switch the former to "detected."

We record the properties of each "detected" transiting planet, as well as its provenance (whether from a dynamically hot or cool configuration). For the sake of comparison, we repeat the exercise with the completeness function of Kepler (Dressing \& Charbonneau 2015), so that we we can directly compare Kepler observables to those predicted for TESS. To generate synthetic $\mathrm{Ke}$ pler systems, we employ a stellar mass $M_{\star}=0.50 M_{\odot}$ as compared to $M_{\star}=0.40 M_{\odot}$ for TESS to reflect a typical $\mathrm{M}$ dwarf from both surveys (we note that altering the central mass by $20 \%$ results in only small changes to the resulting observables).

\section{RESULTS}

We revisit the goals enumerated in Section 1.

\subsection{Summary of Planet Detections}

In Figures 5 and 6, we show the resulting distribution of properties for the $\mathrm{M}$ dwarfs observed by the Kepler (in grey) and TESS missions (in blue). The distributions shown in Figure 5 are normalized to compare directly to the Kepler yield, shown in red, while the distributions in Figure 6 are normalized to one. The transit duration ratio, here denoted as $\xi$, is the one defined by Fabrycky et al. (2012a). For each parameter, we show the mean contribution to the total distribution from the dynamically cooler Population 1 (green) and the dynamically hotter Population 2 (orange). The first immediately noticeable difference is in the period and radius distributions, where the effects of the TESS completeness are clear. TESS will skew heavily toward detecting larger planets than Kepler and at shorter orbital periods. We note that the mutual Hill spacing distribution shown in the second panel of Figure 6 is the true mutual spacing, not the (wider) spacing that would be measured between only detected planets.

In addition to the shapes of these distributions, it's useful to note the raw number of expected host stars and host planets. From the posterior distributions in the modeled number of detections $\mu i, j$, we estimate that the TESS mission will find $990 \pm 350$ planets orbiting $715 \pm 255$ early-to-mid M dwarf host stars. Unsurprisingly, given their strong representation among detected systems, the largest contribution to the uncertainty budget on the number of planets is the uncertainty on the fraction of planetary systems in compact multiples (see Section 3.4).

We also investigate the subset of small, cool planets likely to be prioritized for follow-up with $J W S T$. We define "small" here to be radii $<2 R_{\oplus}$ and "cool" to be periods $20<P<40$ days (approximating the habitable zone of an M4V dwarf). Among the $990 \pm 350$ planets detected by TESS, $15 \pm 7$ meet this criteria. Critically for transit follow-up, an additional $39 \pm 20$ planets in this radius and period range are undetected, but orbit stars for which TESS detected another planet. For the likeliest rocky planets with radii $<1.25 R_{\oplus}$ in the same period range, TESS will detect $2_{-1}^{+2}$ (consistent with the 3 planets with radii $<1.5 R_{\oplus}$ and with orbital periods $>20$ days in the sample published in Sullivan et al. 2015). In even starker contrast with slightly larger planets, $19 \pm 12$ such planets will orbit known TESS hosts, but elude detection by TESS proper. In the hypothetical situation where each known TESS M dwarf host received 40 days of uninterrupted follow-up observation, the yield in newly uncovered temperate Earths would be triple or more that of TESS itself. It stands to reason that follow-up efforts with even moderate sensitivity at longer periods will uncover one or two of these, comparable to the number found in the mission data alone. We describe follow-up implications in greater detail in Section 3.3 .

\subsection{Multiple-transiting systems}

Among these estimated $715 \pm 255 \mathrm{M}$ dwarf planet hosts identified by TESS, the mission will detect at least two planets around $140 \pm 70$ stars. The approximate $20 \%$ contribution of multis to the host star budget is similar to Kepler (see top panel of Figure 5). Even with the steepness of its completeness function with period, we predict the mission will detect $32 \pm 19$ systems with 3 more or transiting planets: a number that makes intuitive sense given the fact that $20 \%$ of mid-M dwarfs host 2 or more planets interior to 10 days, and the average TESS star will receive 27 days of coverage.

Figure 7 shows a representative sample of TESS singles and multis, with a random selection of 20 systems from each population. Black circles, scaled to planet size, depict detections, where red circles are missed planets. The steep TESS radius completeness is especially evident visually here. We have indicated with blue circles the planets that exhibit TTV, assigned from the Xie et al. (2014) occurrence rates as described in Section 2.1, The much higher rate of TTV among multi-transiting systems (even if only one planet was detected) is visually apparent: indeed, with a $3 \%$ occurrence of TTV among singly transiting systems, none ought to appear in such a small representative sample. We note for clarity that we have shown 20 representative singly-transiting and 20 representative multiply-transiting systems as seen by TESS for a sense of their architectures. However, 50:50 is not representative of their relative contributions to the total TESS yield as we describe above.

\subsection{Implications for transit follow-up}

The best-fit ensemble completeness function for TESS in the top left panel of Figure 4 has a critical implication specific to transit follow-up. As a whole, TESS will see a $1.5 R_{\oplus}$ planet $20 \%$ of the time (though the exact completeness depends on the period of the planet as well). Comparing this modest likelihood with the occurrence rates of both Morton \& Swift (2014) and Dressing \& Charbonneau (2015) (both of which show planets peaking in occurrence at 1-1.5 $R_{\oplus}$ ), it's clear that the majority of planets orbiting $\mathrm{M}$ dwarfs will be 

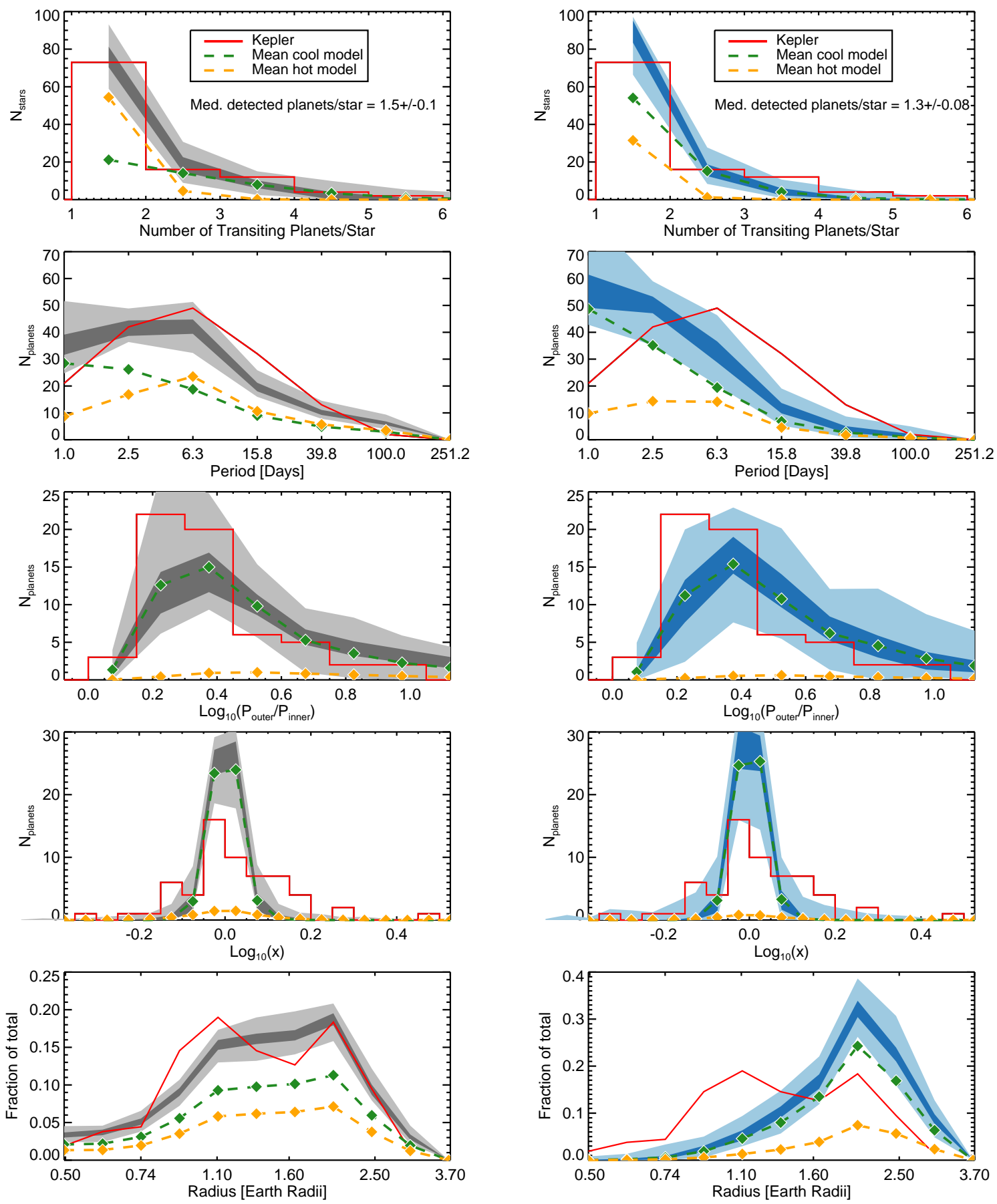

FIG. 5.- Resulting posterior distributions predicted for the Kepler mission at left (grey) and TESS mission at right. In order from top to bottom: number of detected transiting planets per star, periods of detected planets, period ratio between adjacent observed transiting planets, velocity-normalized transit duration ratio between adjacent planets, and planetary radius. All distributions have been normalized to compare to the shape of the actual observed Kepler distributions in red. 

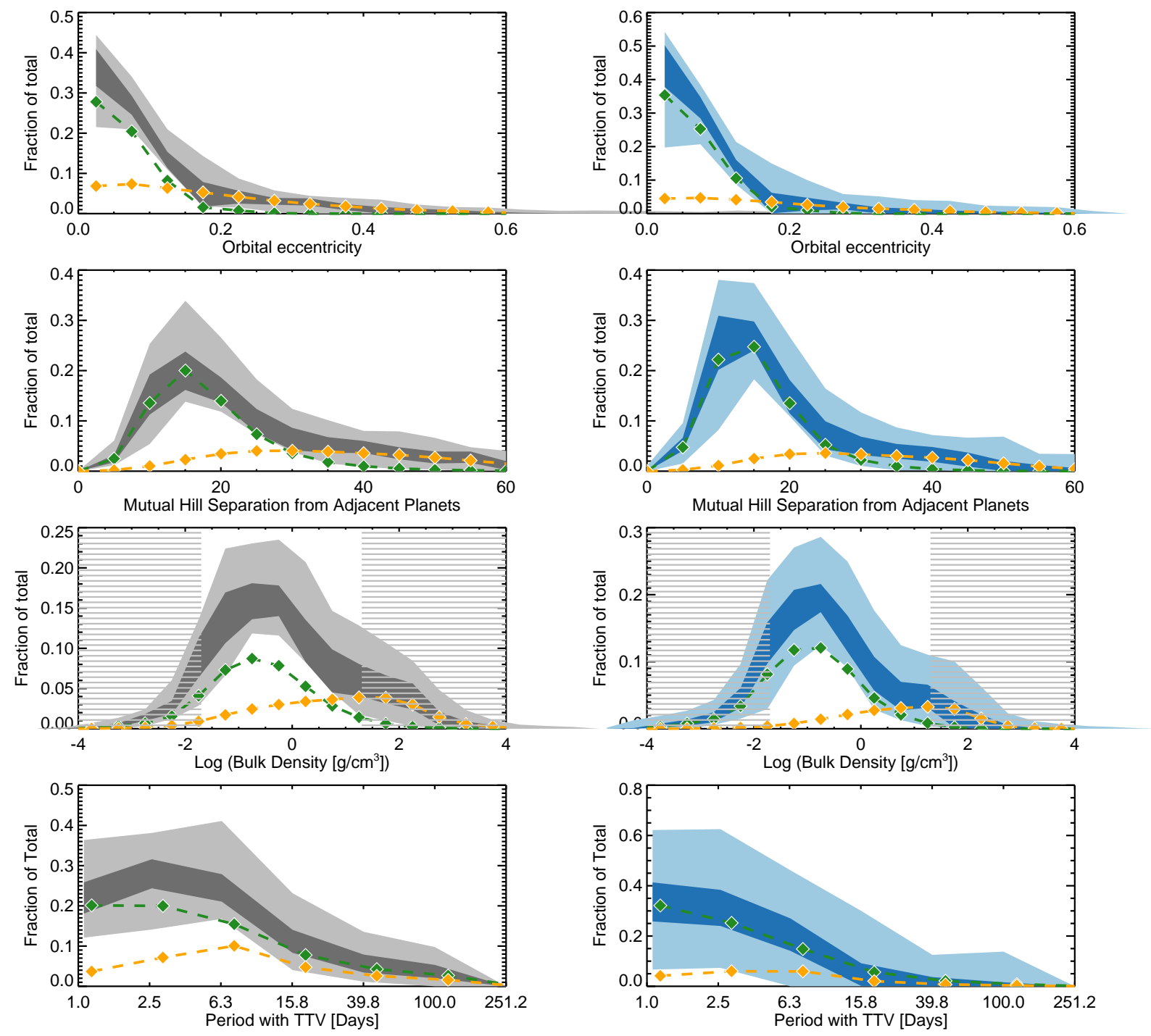

FIG. 6.- Resulting posterior distributions predicted for the Kepler mission at left (grey) and TESS mission at right. In order from top to bottom: orbital eccentricity of detected planets, mutual Hill spacing from neighboring planets, predicted density from Hill spacing per Dawson et al. (2016), and periods of planets showing transit timing variations

missed. Yet, the majority of stars around which TESS finds a planet will host a compact multiple system (primarily because of the steep period completeness function). For systems of 3 or more transiting planets, $40 \%$ of the time TESS will detect only one planet, typically the very largest. This means that missed planets orbiting known TESS hosts will be remarkably common. We quantify this result in Figure 8, showing the number of missed planets per 200 TESS host stars (that is, stars for which TESS detected one or more planets). Among 200 TESS host stars, typically 250 planets will be detectable in the mission light curves themselves. But on average, half that number lurk below the mission sensitivity: $116_{-28}^{+28}$ planets per 200 host stars. This is also visibly apparent in Figure 7 where red (missed) planets are common among their detected (black) neighbors.

Therefore, follow-up efforts sensitive to planets < $1.5 R_{\oplus}$, even those with very modest period completeness, will readily find additional planets. For example, a hypothetical survey of 200 TESS hosts, sensitive to $1 R_{\oplus}$ planets, with $100 \%$ completeness out to only 2.2 days, will find an average of 11 additional planets (at least 6 , and as many as 16 , within the $68 \%$ confidence interval). Especially promising for transit follow-up efforts: there are enough missed planets that surveys with $25 \%$ completeness at 40 days can expect to find at least one rocky $\left(1-1.5 R_{\oplus}\right.$ ) planet in its habitable zone (for an M4V dwarf).

We point out the subtle but important distinction between number of planets missed per 200 host stars and the number of those stars that host at least one missed planet. Among 200 hosts to at least one transiting planet, on average 120 host only that transiting planet. Among the remaining 80 hosts: 50 host one missed planet, 20 host 2 missed planets, and 10 host 3 or more. The odds are statistically distinct for hosts to one TESS-detected planet versus multiple detected planets, and also hosts to planets with detected transit-timing 

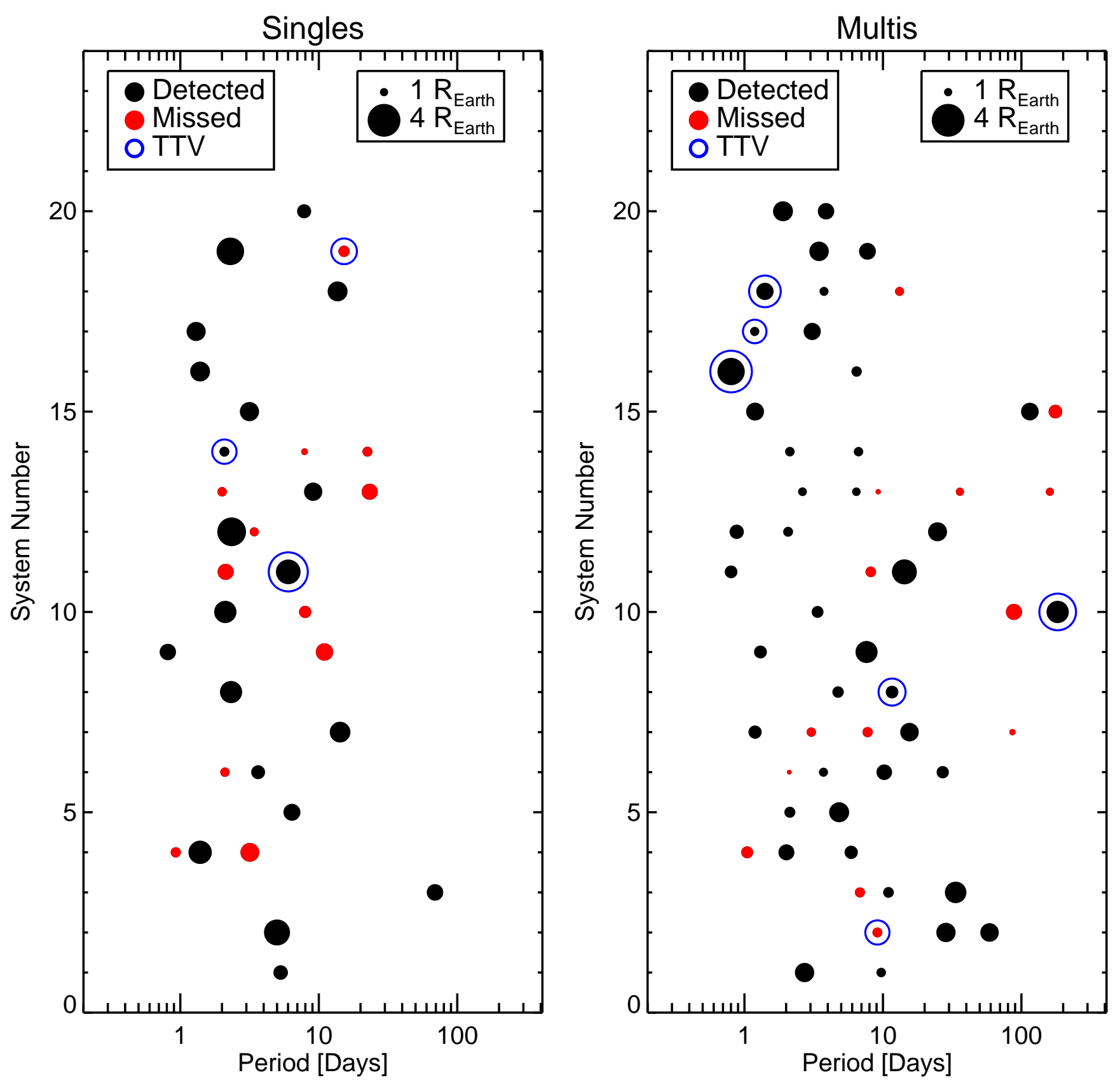

FIG. 7.- Left panel: A representative sample of M dwarf planetary systems in which TESS detected a single transiting planet. We depict only the transiting planets orbiting each star. Black indicates TESS-detected planets, while red indicates planets that were missed. Planet radii is show by the relative sizes of circles, while planets exhibiting TTV are ringed in blue. Left panel: A simple sample for systems in which TESS detected two or more transiting planets.

variations. For the sake of illustration, among a sample of 200 systems where TESS detected at least 2 transiting planets, now on average 95 host at least one additional transiting planet. And among a sample of 200 systems with a TESS-detected planet that also exhibits TTV, the odds are yet more favorable: 112 hosts out of 200 would have additional unseen planets among them.

\subsection{Underlying Occurrence Rate}

We have employed the posterior distribution in $f$ from Ballard \& Johnson (2016), where $f$ in that work is the fraction of transiting systems in the compactmultiple configuration. However, because selection bias favors the detection of these systems, they are overrepresented among the Kepler host star sample compared to their true underlying fraction among stars. We aim to test for consistency with occurrence rate for compact multiples orbiting $\mathrm{M}$ dwarfs derived by Muirhead et al. (2015), though we approach the problem in different ways. Firstly, the definition of "compact multiple" in Muirhead et al. (2015) is 2 or more planets interior to a 10 day orbit. Practically speaking, if planets are 
Number of missed planets per 200 TESS host stars
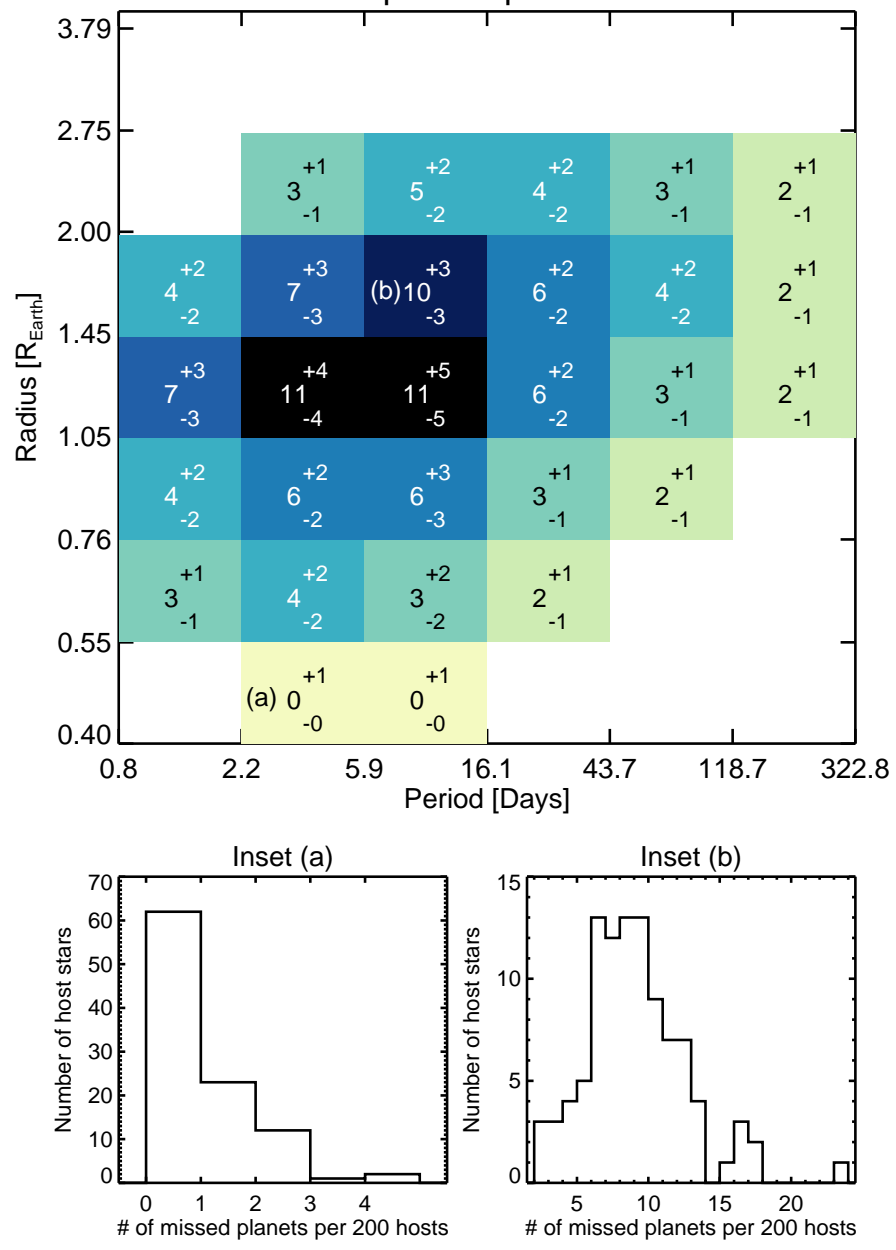

FIG. 8.- Top panel: For every 200 TESS host stars, the number of transiting planets below TESS' detection threshold as a function of planetary radius and period.Bottom panels: The Poisson distributions in number of missed planets per 200 host stars for two example bins, indicted by (a) and (b) in the top panel.

spaced equally in log semi-major axis, on average 5 $M_{\oplus}$, and dynamically stable, this corresponds to systems with 7 or more planets interior to 200 days. This value is safely within the posterior distribution of number of planets per star $N_{1}$ found by Ballard \& Johnson (2016): $40 \%$ of the distribution lies at 7 planets/star or greater. Secondly, Muirhead et al. (2015) employed inverse-detection-efficiency machinery and compared to the number of stars hosting 2 or more planets compared to the number hosting no planets. In comparison, in Ballard \& Johnson (2016), we ignored entirely systems hosting no transiting planets. We employed forward modeling to compare models to a different observable altogether: the shape of the distribution in the number of transiting planets per star. We invert the posterior in $f$ to $f_{\star}$ as follows.

For each $10^{4}$ planetary systems we generate from the posterior on $f$ (described in Section 2.2. we solve empirically for the fraction of stars $f_{\star}$ in Population 1 . We make the assumption that every $\mathrm{M}$ dwarf in the sample hosts a planetary system of some kind, whether in Populations 1 or 2 , so that the fractions sum to one. This assumption
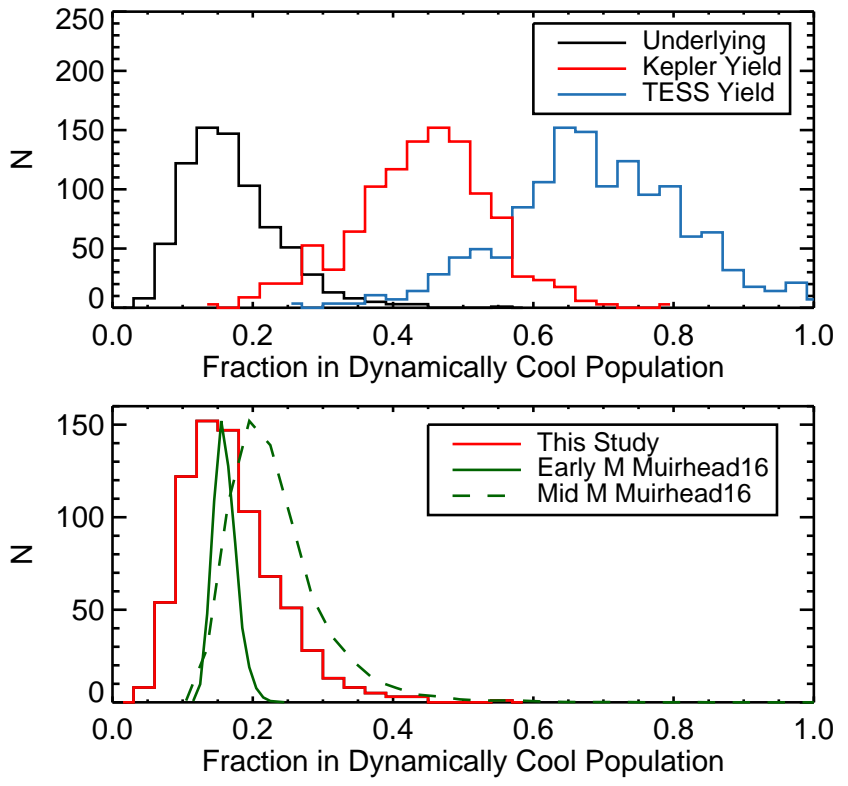

Fig. 9.- Top panel: Fractions of systems in the compactmultiple configuration. Bottom panel: Same population of detected Sullivan et al. (2015) planets, now with models for predicted planet detections overplotted in gray.

brings consistency between the mean number of planets per star of 2.0-2.5 determined by Morton \& Swift (2014) and Dressing \& Charbonneau (2015), and our planetary mixture model in which some stars host 5 planets and others host 1, as we described in Ballard \& Johnson (2016). We record this fraction $f_{\star}$ at each step of the MCMC chain. The lower panel of Figure 9 shows the resulting distribution in $f_{\star}$, as compared to results from Muirhead et al. (2015). While compact multiples make up $45 \pm 10 \%$ of transiting systems found by Kepler, they are only $15 \pm 5 \%$ of all planetary systems orbiting early $\mathrm{M}$ dwarfs. This is consistent with the $15.9 \pm 1.5 \%$ found by Muirhead et al. (2015) for early M dwarfs.

We compare in Figure 9 the resulting distributions in $f_{\star}$ between the Kepler and TESS missions. The selection bias that favored the detection of compact multiples from the Kepler Mission is still greater for NASA's TESS mission. We show in the top panel of Figure 9 the fraction of compact multis within the sample of planet hosts for both Kepler and TESS. Now the fraction $f$ is $68 \pm 12 \%$, showing that the steep period completeness for TESS will likely result in $5 \mathrm{x}$ the rate of compact multiples among TESS hosts than the underlying rate in nature.

\subsection{Implications for ensemble eccentricity}

Orbital eccentricity in $\mathrm{M}$ dwarf planetary systems has complicated implications for habitability. For the smallest stars, even a modest eccentricity can induce a sterilizing "runaway greenhouse" effect (Barnes et al. 2013). On the other hand, modest eccentricity may be sufficient to induce plate tectonics in the absence of radiogenic heating Jackson et al. (2008). The eccentricity of a planet also shapes how we interpret its atmospheric signature (for a detailed summary, see Shields et al. 2016). 


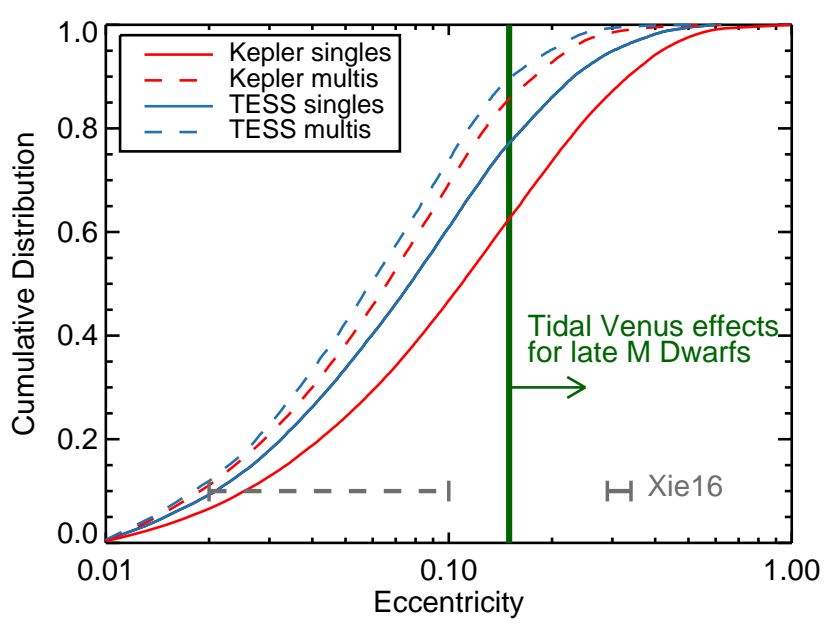

FIG. 10. - The predicted cumulative eccentricity distribution for both TESS (blue) and Kepler (red) missions, where planets in a system with a single detected planet (solid line) are shown separately from planets in systems with multiple detected planets (dashed line). We have overplotted in gray the empirical eccentricites measured for Kepler's singles (dashed) and multis (solids) from Xie et al. (2016) for comparison.

TESS' strong selection bias for shorter periods favors the discovery of compact and generally dynamically cooler systems generally, which we quantify in the previous section. This is particularly true for the multipleplanet systems uncovered by TESS, whose membership is almost certainly in this population. Figure 10 summarizes this result, showing the cumulative eccentricity distributions for both the single and multiple transiting systems. A comparison between the TESS and Kepler distribution shows the predicted lower eccentricity for TESS planetary systems on average. This effect is strongest for the multi-transit systems from TESS, for which $80 \%$ of planets have orbital eccentricities less than 0.1 . We overplot the empirical result of Xie et al. (2016) for the Kepler singles and multiples, measured from photometry. The eccentricities inferred for Kepler singles from this study are lower than the average measurement from Xie et al. (2016). However, we consider here the subset of M dwarfs rather than the full Kepler sample examined by Xie et al. (2016).

We overplot, for the sake of comparison, an eccentricity associated with runaway tidal heating on late $\mathrm{M}$ dwarfs from Barnes et al. (2013). There exists a range of cutoff eccentricities for this effect, depending upon bulk planet composition, atmospheric composition, and assumptions about exactly how the dynamical heating occurs. For this reason, the cutoff shown here is illustrative rather than definitive. We have depicted the cutoff eccentricity of 0.15 for the sake of comparison with the cumulative eccentricity distributions. For higher orbital eccentricities, planets $1 M_{\oplus}$ and larger in the habitable zone of $0.25 M_{\odot}$ stars are predicted to experience a runaway Venus effect (Barnes et al. 2013). We note that TESS planets are safer from this effect on average, with the planets in multi-planet systems safest (with only $10 \%$ possessing orbital eccentricities greater than 0.15).

\subsection{Transit-timing Variations}

While TESS itself may only rarely have the observational baseline to observe transit-timing variations, we can see predict their frequency among TESS-detected planets. Employing the empirical TTV likelihood as a function of number of transiting planets from Xie et al. (2014), we predict the TTV likelihood among the TESS transiting planets. Figure 11 shows a comparison of the TTV fraction for both missions. In the Kepler sample, the overall rate of $5 \%$ reflects the mixture of planetary systems to which compact multis contribute only half. We show the TTV occurrence fraction from both populations in green (dynamically cool) and orange (dynamically hot), where the height of the histogram reflects the contribution of that population to the total number of planets. The TESS completeness, in contrast, heavily favors the types of compact multiples that exhibit TTV. For TESS, these types of planetary systems will comprise a likely $70 \%$ of the yield, as we describe in the previous section. The fact that the final TTV rate is similar to Kepler's is due to a subtlety. Though compact multis are favored for detection by TESS, only one or two planets are typically detected in these systems, even if 3 or 4 transit. In comparison, consider the 3 and 4 transiting planets systems detected by Kepler. With the higher TTV probability per planet, the fact that there are 3 or 4 planets each with this higher probability (as opposed to 1 or 2) skews the overall TTV likelihood higher. The tradeoff between these two phenomena results in a TTV fraction similar to Kepler's, despite TESS' strong preference for compact multiples in which TTV are more common.

\subsection{Planet Bulk Density}

The third panel of Figure 6 shows the posterior distribution in theoretical density assigned from mutual Hill spacing per Dawson et al. (2016). Only planets in systems for which Hill spacing is applicable (those with with 2 or more planets) contribute to this distribution. We have indicated with cross-hatching the densities too high or low to be included in the Dawson et al. (2016) metric. The higher fraction of compact multiples within TESS, and their accompanying close orbital spacing, maps to a predicted lower density on average. Drawing from the entire predicted TESS yield for $\mathrm{M}$ dwarf systems with 2 or more planets, we find $\bar{\rho}=0.3_{-0.1}^{+0.3}$. which are likely $70 \%$ of the TESS planetary systems. The corresponding mean density for Kepler systems with 2 or more planets is $\bar{\rho}=0.9_{-0.4}^{+0.6}$. This prediction for the average fluffiness of TESS planets will ultimately be tested with radial velocity follow-up and transmission spectroscopy: we leave specific implications for those follow-up efforts for future work.

\section{SUMMARY AND CONCLUSIONS}

Using the injected and detected samples published by Sullivan et al. (2015), we have extracted a completeness function with planet radius and orbital period for TESS $\mathrm{M}$ dwarfs. We first demonstrate that the application of this completeness function to the Sullivan et al. (2015) injected planet sample correctly recovers the planet detections from that work. We then re-apply the completeness function, assuming a different planet occurrence rate. Rather than assuming 2-3 planets per star, 

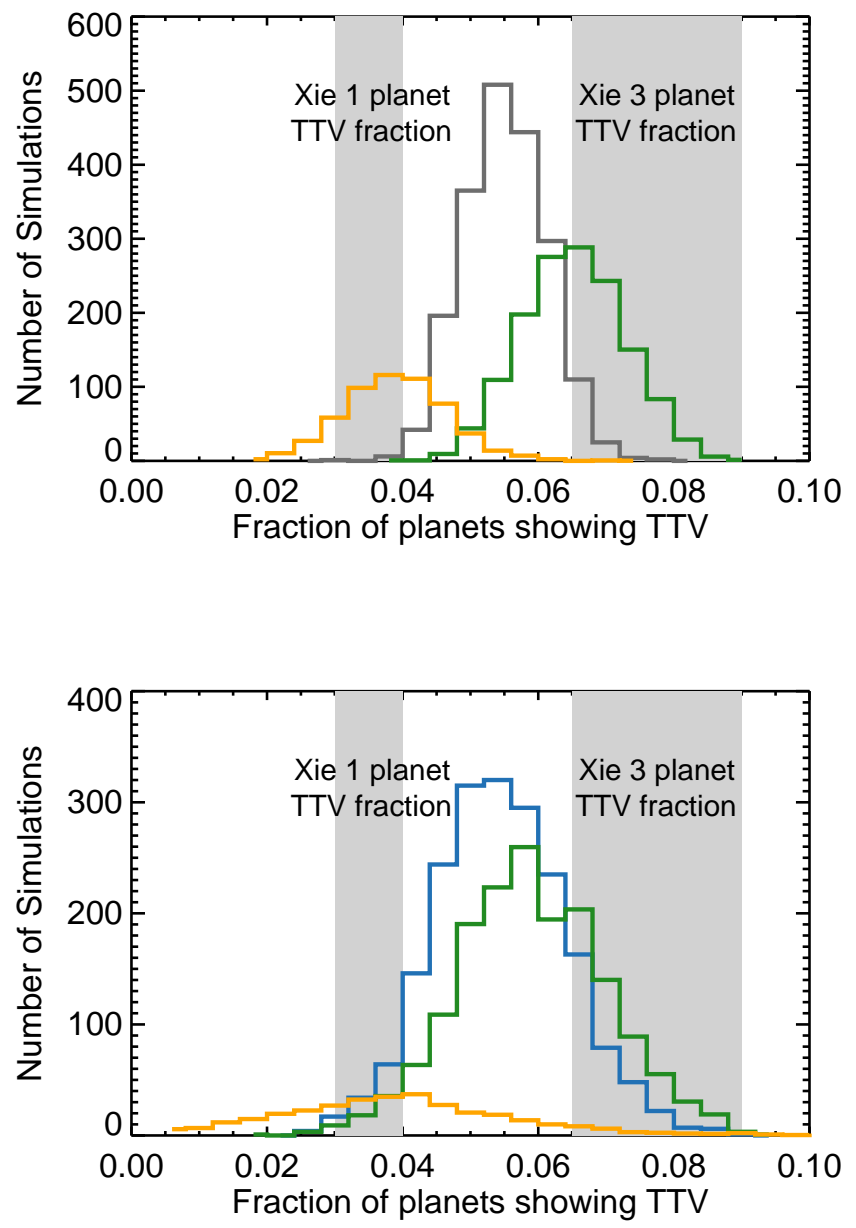

FIG. 11. - Top panel: Posterior distribution for fraction of $\mathrm{Ke}$ pler planets (black) exhibiting transit timing variations, as defined by "Case 3" in Xie et al. (2014). The separate distributions for the dynamically cool (green) and dynamically hot (orange) populations have been scaled to reflect their relative contributions to the number of planets. Bottom panel: The same distributions predicted for the TESS sample.

we assume a mixture model with two types of planetary systems. One type contains $>5$ closely aligned planets (around 20\% of stars, per Section $\$ 3.4$ ), and one that contains one planet, or two planets with high mutual inclination respective to one another. We return to our enumerated list of goals from Section 1, to summarize our findings on each.

1. We predict that TESS will uncover $990 \pm 350$ planets orbiting $715 \pm 255$ stars, a factor of 1.5 more than predicted in Sullivan et al. (2015) (§3.1). The error budget on number of detections is dominated by uncertainty on the underlying fraction of compact multiples in nature.

2. Even given the typical duration of 27 days per star, we predict TESS will detect two or more planets around $140 \pm 70$ stars among the hosts above. The approximate $20 \%$ contribution of multis to the total host star budget is similar to Kepler M dwarfs. The high rate of compact multiples indicates that
TESS will even detect three or more planets around $32 \pm 19$ stars (§3.2).

3. Among 200 typical TESS M dwarf host stars, an average of 250 planets will be detectable in the mission data itself. We predict half that number lurk below the mission sensitivity: $116_{-28}^{+28}$ planets per 200 host stars. Many of these planets will be readily detectable from ground-based surveys and space-based campaigns $(\S 3.3)$

4. We confirm the compact multiple rate (defined as 2 or more planets with orbital periods $<10$ days) among $\mathrm{M}$ dwarfs measured previously in the literature. We find this rate to be $15 \pm 5 \%$ among early M dwarfs, as compared to $15.9 \pm 1.5 \%$ (Muirhead et al. 2015), using a different technique. While compact multiple hosts are not the majority in nature, the relative ease of their detection makes them overrepresented in transit surveys: they are $45 \pm 10 \%$ of Kepler-detected planet hosts, and we predict they will be $68 \pm 12 \%$ of TESS-detected planet hosts.

5. By virtue of the lower average eccentricities of planets in multiple-planet systems, we predict that average orbital eccentricity of planets detected by $T E S S$ will correspondingly be lower than the average for Kepler. For systems in which TESS detects two or more transiting planets, $80 \%$ of planets will have orbital eccentricities less than 0.1

6. Despite the higher fraction of compact multiples in the TESS yield, the number of planets detected by TESS that exhibit transit timing variations (as defined by Xie et al. 2014) will be similar to the overall $5 \%$ observed for Kepler. These TTV will not be detectable by TESS itself as for Kepler, but we predict the underlying rate among detected planets to be similar.

7. Employing the planet formation theory of Dawson et al. (2016) linking adjacent planet spacing to planet bulk density, we predict that TESS planets will be fluffier on average. We apply this metric to systems with at least two planets, to find $\bar{\rho}=0.3_{-0.1}^{+0.3}$ among TESS planets, as compared to $\bar{\rho}=0.9_{-0.4}^{+0.6}$ for Kepler planets. $(\S 3.7)$

We conclude by re-emphasizing the ground and spacebased opportunity for photometric follow-up, specific to planet discovery. Around stars for which TESS detects one or more planets, we predict a wealth of additional transiting planets: present but undetectable in the TESS light curves (Section §3.3). We take as an example a hypothetical survey of 200 TESS host stars, sensitive to 1 $R_{\oplus}$ planets and with $100 \%$ completeness out to only 2.2 days. Such an study will detect $11 \pm 5$ additional planets. Odds improve yet more if TESS has detected two or more planets around the star, or if one of the planets exhibits transit-timing variations. An extended Spitzer mission (as detailed by Yee et al. 2017) would have the photometric sensitivity time baseline to be sensitive to rocky planets even in the habitable zone of TESS hosts. While we predict TESS itself to detect $2_{-1}^{+2}$ planets $¡ 1.25 R_{\oplus}$ with 
orbit periods $20<P<40$ days, an additional $19 \pm 12$ such planets will orbit known TESS hosts to another transiting planet, but elude detection by TESS proper. An extended Spitzer mission may be singularly suited for their discovery.

The soon-to-be-launched CHaracterising ExOPlanet Satellite (CHEOPS, Fortier et al. 2014) will gather high-precision transit observations to constrain (among many objectives) planetary atmospheres and formation. The telescope, in a geocentric orbit, will continuously point at a single target for a typical 612 hours; however, it can achieve stares of a few weeks' duration (Broeg et al. 2013), and in principle could also readily detect additional planets. Ad- ditionally, ground-based photometric surveys such as MEarth (Nutzman \& Charbonneau 2008) and TRAPPIST (Gillon et al. 2011) have already demonstrated the ability to detect planets $<2$ orbiting $\mathrm{M}$ dwarfs (Charbonneau et al. 2009; Berta-Thompson et al. 2015; Dittmann et al. 2017; Gillon et al. 2017). The forthcoming wealth of TESS planets portends a strong synergy with follow-up efforts.

\section{ACKNOWLEDGMENTS}

We thank Philip Muirhead, Joshua Winn, and particularly John Johnson for discussions that immeasurably improved this manuscript. S.B. is funded by the MIT Torres Fellowship for Exoplanet Science.

\section{REFERENCES}

Ballard, S., \& Johnson, J. A. 2016, ApJ, 816, 66

Barnes, R., Mullins, K., Goldblatt, C., Meadows, V. S., Kasting, J. F., \& Heller, R. 2013, Astrobiology, 13, 225

Berta-Thompson, Z. K., et al. 2015, Nature, 527, 204

Bonfils, X., et al. 2013, A\&A, 549, A109

Bovaird, T., \& Lineweaver, C. H. 2017, MNRAS, 468, 1493

Bowler, B. P., Liu, M. C., Shkolnik, E. L., \& Tamura, M. 2015, ApJS, 216, 7

Broeg, C., et al. 2013, in Hot Planets and Cool Stars, ed. R. Saglia, Vol. 47 (Garching, Germany: EPJ Web of Conferences)

Buchner, J., et al. 2014, A\&A, 564, A125

Charbonneau, D., et al. 2009, Nature, 462, 891

Christiansen, J. L., et al. 2016, ApJ, 828, 99

Clanton, C., \& Gaudi, B. S. 2016, ApJ, 819, 125

Dawson, R. I., Lee, E. J., \& Chiang, E. 2016, ApJ, 822, 54

Dittmann, J. A., et al. 2017, Nature, 544, 333

Dressing, C. D., \& Charbonneau, D. 2013, ApJ, 767, 95

-. 2015, ApJ, 807, 45

Fabrycky, D. C., et al. 2012a, ApJ, 750, 114

—. 2012b, submitted to ApJ (arXiv: 1202:6328)

Feroz, F., \& Hobson, M. P. 2008, MNRAS, 384, 449

Feroz, F., Hobson, M. P., \& Bridges, M. 2009, MNRAS, 398, 1601

Feroz, F., Hobson, M. P., Cameron, E., \& Pettitt, A. N. 2013, ArXiv e-prints

Fortier, A., Beck, T., Benz, W., Broeg, C., Cessa, V., Ehrenreich, D., \& Thomas, N. 2014, in Proc. SPIE, Vol. 9143, Space Telescopes and Instrumentation 2014: Optical, Infrared, and Millimeter Wave, 91432J

Gaidos, E., Mann, A. W., Kraus, A. L., \& Ireland, M. 2016, MNRAS, 457, 2877

Gardner, J. P., et al. 2006, Space Sci. Rev., 123, 485

Gillon, M., Jehin, E., Magain, P., Chantry, V., Hutsemékers, D., Manfroid, J., Queloz, D., \& Udry, S. 2011, in European Physical Journal Web of Conferences, Vol. 11, European Physical Journal Web of Conferences, 06002

Gillon, M., et al. 2017, Nature, 542, 456

Hawkins, D. M. 1994, Computational Statistics, 9, 2330247
Howard, A. W., et al. 2012, ApJS, 201, 15

Jackson, B., Greenberg, R., \& Barnes, R. 2008, ApJ, 678, 1396

Johnson, J. A., Aller, K. M., Howard, A. W., \& Crepp, J. R. 2010, PASP, 122, 905

Limbach, M. A., \& Turner, E. L. 2015, Proceedings of the

National Academy of Science, 112, 20

Lissauer, J. J., et al. 2011, ApJS, 197, 8

Mills, S. M., \& Mazeh, T. 2017, ApJ, 839, L8

Montet, B. T., Crepp, J. R., Johnson, J. A., Howard, A. W., \& Marcy, G. W. 2014, ApJ, 781, 28

Moriarty, J., \& Ballard, S. 2016, ApJ, 832, 34

Morton, T. D., \& Swift, J. 2014, ApJ, 791, 10

Morton, T. D., \& Winn, J. N. 2014, ApJ, 796, 47

Muirhead, P. S., Dressing, C., Mann, A. W., Rojas-Ayala, B., Lepine, S., Paegert, M., De Lee, N., \& Oelkers, R. 2017, ApJ, submitted (arXiv:1710.00193)

Muirhead, P. S., et al. 2015, ApJ, 801, 18

Mulders, G. D., Pascucci, I., \& Apai, D. 2015, ApJ, 814, 130

Murray, K., Müller, S., \& Turlach, B. A. 2013, Computational Statistics, 28, 1989

Nutzman, P., \& Charbonneau, D. 2008, PASP, 120, 317

Ricker, G. R., et al. 2014, in Proc. SPIE, Vol. 9143, Space Telescopes and Instrumentation 2014: Optical, Infrared, and Millimeter Wave, 914320

Rogers, L. A. 2015, ApJ, 801, 41

Shields, A. L., Ballard, S., \& Johnson, J. A. 2016, Physics Reports, 663, 1

Sullivan, P. W., et al. 2015, ApJ, 809, 77

Tarter, J. C., et al. 2007, Astrobiology, 7, 30

Turlach, B. A., \& Murray, K. 2016, MonoPoly: Functions to Fit Monotone Polynomials, $\mathrm{r}$ package version 0.3-8

Wolfgang, A., Rogers, L. A., \& Ford, E. B. 2016, ApJ, 825, 19

Xie, J.-W., et al. 2016, Proceedings of the National Academy of Science, 113, 11431

Xie, J.-W., Wu, Y., \& Lithwick, Y. 2014, ApJ, 789, 165

Yee, J. C., et al. 2017, ArXiv e-prints

Zeng, L., \& Jacobsen, S. B. 2017, ApJ, 837, 164 